\title{
Investigation of two-dimensional acoustic resonant modes in a particle separator
}

\author{
R.J. Townsend ${ }^{\mathrm{a}}$, M. Hill ${ }^{\mathrm{a}, *}$, N.R. Harris ${ }^{\mathrm{b}}$, N.M. White ${ }^{\mathrm{b}}$ \\ ${ }^{a}$ School of Engineering Sciences, University of Southampton, SO17 1BJ, UK \\ ${ }^{\mathrm{b}}$ Electronics and Computer Science, University of Southampton, SO17 1BJ, UK
}

Available online 2 June 2006

\begin{abstract}
Within an acoustic standing wave particles experience acoustic radiation forces, a phenomenon which is exploited in particle or cell manipulation devices. When developing such devices, one-dimensional acoustic characteristics corresponding to the transducer(s) are typically of most importance and determine the primary radiation forces acting on the particles. However, radiation forces have also been observed to act in the lateral direction, perpendicular to the primary radiation force, forming striated patterns. These lateral forces are due to lateral variations in the acoustic field influenced by the geometry and materials used in the resonator. The ability to control them would present an advantage where their effect is either detrimental or beneficial to the particle manipulation process.

The two-dimensional characteristics of an ultrasonic separator device have been modelled within a finite element analysis (FEA) package. The fluid chamber of the device, within which the standing wave is produced, has a width to height ratio of approximately 30:1 and it is across the height that a half-wavelength standing wave is produced to control particle movement. Two-dimensional modal analyses have calculated resonant frequencies which agree well with both the one-dimensional modelling of the device and experimentally measured frequencies. However, these two-dimensional analyses also reveal that these modes exhibit distinctive periodic variations in the acoustic pressure field across the width of the fluid chamber. Such variations lead to lateral radiation forces forming particle bands (striations) and are indicative of enclosure modes.

The striation spacings predicted by the FEA simulations for several modes compare well with those measured experimentally for the ultrasonic particle separator device. It is also shown that device geometry and materials control enclosure modes and therefore the strength and characteristics of lateral radiation forces, suggesting the potential use of FEA in designing for the control of enclosure modes in similar particle manipulator devices.
\end{abstract}

(C) 2006 Elsevier B.V. All rights reserved.

Keywords: Lateral radiation force; FEA; Particles

\section{Introduction}

The action of acoustic radiation forces on particles has been exploited in devices designed to manipulate particles. Such devices include particle separators and fractionators in which the nature of the acoustic field significantly influences the performance of the device. Therefore, in order to design ultrasonic devices reliably, the characteristics of the acoustic field must be considered.

\footnotetext{
${ }^{*}$ Corresponding author. Tel.: +44 23 80593075; fax: +44 2380593053. E-mail address: m.hill@soton.ac.uk (M. Hill).
}

These devices typically rely on the presence of a plane standing wave generated using a plate transducer. The resulting acoustic field is usually described one-dimensionally with the field varying in a direction normal to the transducer plate. Acoustic radiation forces result from these variations, and in a direction normal to the transducer. When the standing wave is generated within a fluid cavity containing a particle suspension, the particles experience an acoustic radiation force which depends on both the density and compressibility of the particles, allowing neutrally buoyant particles to be manipulated and filtered. This will typically move solid phase particles to the pressure 


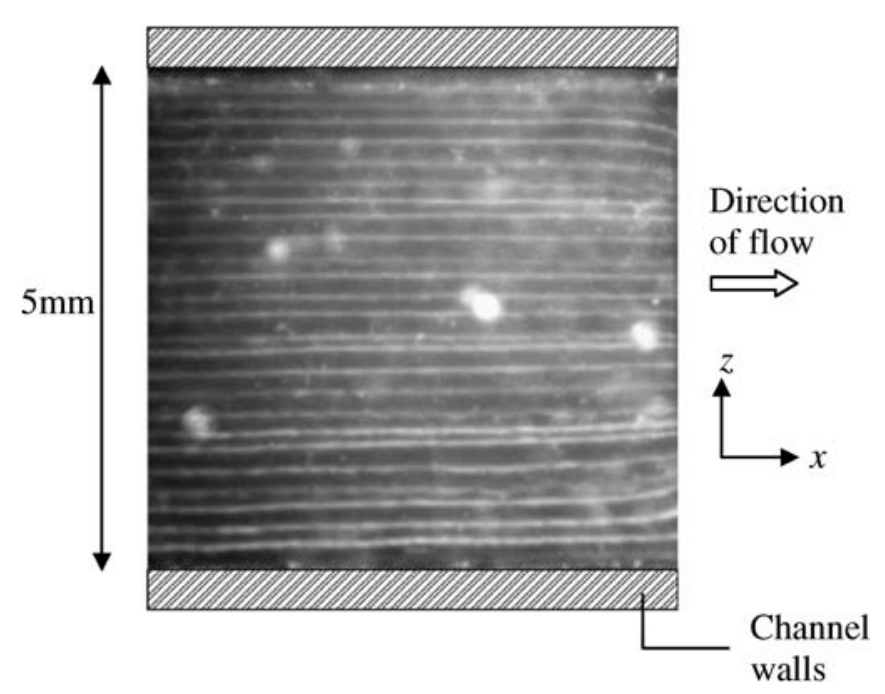

Fig. 1. Striated patterns observed using yeast for conditions of continuous flow-through.

node(s) and form a layer of concentrated particles. However, lateral movement of particles is frequently observed as variations within the acoustic field exist with the particles moving within the nodal plane to areas of high acoustic kinetic energy [1]. The lateral movement of particles can be attributed to lateral variations within the acoustic field which give rise to lateral acoustic radiation forces. The source of these variations is the subject of this paper.

Fig. 1 shows the effect of lateral forces on particles within a particle separation device. The image is taken looking down upon the device and in the axial $(y)$ direction with the transducer located on the underside of the device and orientated in the $x z$ plane. A half-wavelength standing wave is generated within a fluid layer and forms a pressure node along its centre plane, towards which particles move due to axial radiation forces. Within this image the effect of additional lateral forces can be seen where particles have moved to positions of high acoustic kinetic energy and have formed a striated pattern. This reveals the effect of the spatially averaged $z$ component of the radiation force.

Depending on the manipulation process, the presence of these lateral forces can be detrimental to performance and may cause particles to form large aggregates which may sediment out of the suspension or block and disrupt the movement of the fluid. However, some recent devices have demonstrated that lateral forces can be used to advantage by influencing the movement and trapping of particles $[2,3]$.

\section{Enclosure modes within separator device}

The presence of lateral radiation forces are investigated for a micro-engineered particle separator. This device relies on the movement of particles to a nodal plane under the influence of axial $(y)$ radiation forces, then allowing particle clarified flow to be drawn off one side of the particle stream. The device has a layered structure and includes a silicon matching layer, a fluid layer and a Pyrex reflector layer [4]. The layer thickness $y$ dimensions are relatively small compared to the length and width ( $x$ and $z$ dimensions), therefore, when driving the device at frequencies which excite a thickness resonance the acoustic characteristics through the $y$-axis should predominate. Fig. 2 illustrates the basic construction and operation of the device, and orientation of the axial $y$ and lateral $z$ axes.

Lateral variations within the device can be caused by structural modes within the chamber walls, near-field effects or enclosure modes. Here, enclosure modes, which may be excited together with the axial modes required for operation, are investigated in isolation. The expression below describes the enclosures modes for a rectangular cavity with either rigid or pressure release boundaries [5]:

$f=\frac{c}{2} \sqrt{\left\langle\frac{l}{L_{x}}\right\rangle^{2}+\left\langle\frac{m}{L_{y}}\right\rangle^{2}+\left\langle\frac{n}{L_{z}}\right\rangle^{2}}$,

where the various dimensions of the waveguide are given by $L_{x}, L_{y}$ and $L_{z}$ with relative mode orders of $l, m$ and $n$. The existence of such modes within the separator device will give rise to lateral $(z)$ as well as axial $(y)$ pressure gradients and, therefore, components of acoustic force acting in both these directions. Whilst the mode shape and resonant frequency in a simple rectangular cavity can be determined analytically (Eq. (1)), the geometry and therefore the

(a)
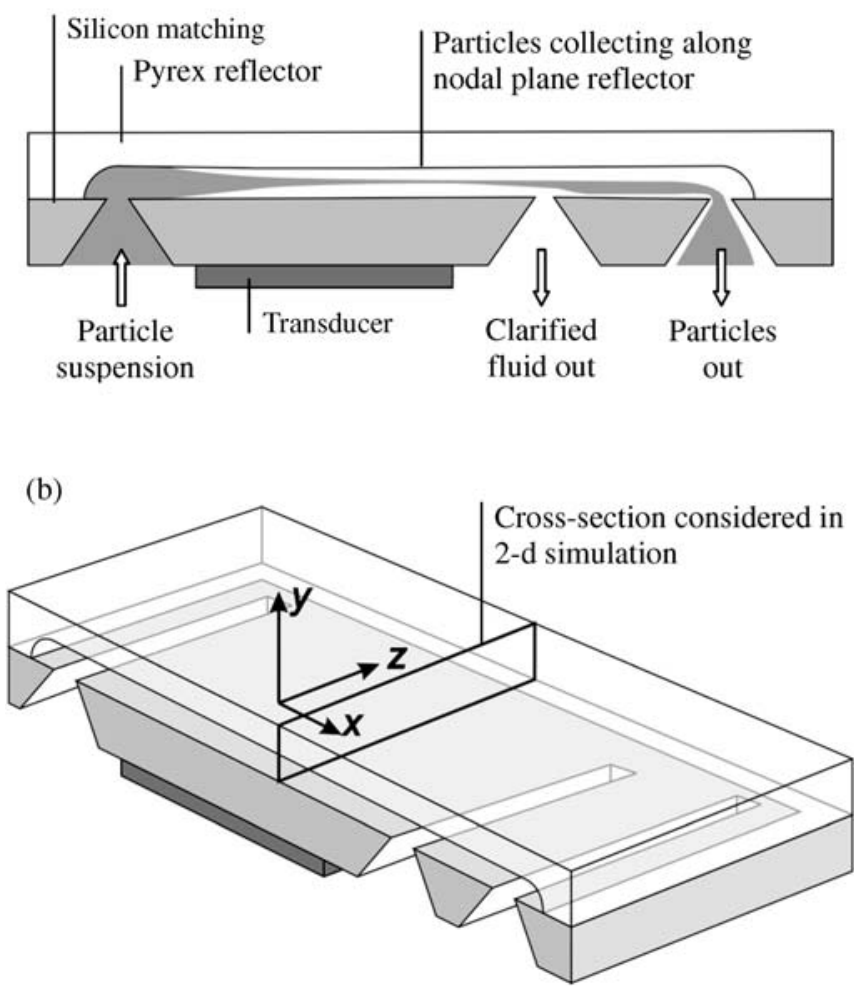

Fig. 2. Schematics of particle separator showing (a) movement of particles through the device and (b) orientation of axial $(y)$ and lateral $(z)$ components. 
mode shapes in layered ultrasonic devices is more complex and a finite element approach is used.

\section{Acoustic simulation}

One-dimensional simulation of the through-thickness (y) mode shape within the fluid and reflector layers is typically used in the design of the separator. For this onedimensional simulation an acoustic impedance transfer model is used which is coded using MATLAB [6,7]. It is also possible to use finite element (FE) analysis to simulate the one-dimensional case for which the computer package ANSYS is used. The FE model includes elements to describe the fluid and reflector layers with a pressure release boundary applied to the external face of the reflector layer. ANSYS 'fluid' elements with density and sonic velocity properties are used as their output contain acoustic field properties and allow the simulation of acoustic enclosure modes, rather than structural modes which are not the subject of this paper. A comparison of the MATLAB model and FE modal analysis is shown in Fig. 3 and shows $\mathrm{a} \sim \lambda / 2$ mode in the fluid layer which will cause suspended particles to move to the nodal plane. The difference between the two curves may be due to the different methods used to describe losses in the respective models. This mode occurs at a frequency of $\sim 3.4 \mathrm{MHz}$ in the fabricated test device, therefore both models are thought to present good estimates of the axial field.

To predict the presence of lateral variations in the acoustic field, the FEA model is extended to describe the fluid and reflector layers in two-dimensions and a section across the width of the device is simulated (Fig. 2(b)). Again, modal analyses are used in which mode shapes and the associated resonant frequencies are calculated. The simulation is based on the measured width of the Pyrex layer (external width) of $6.8 \mathrm{~mm}$ and design width of the fluid cavity of $5 \mathrm{~mm}$. The curved side-walls created by an isotropic etch process are also included in the model. This model can be compared to experimental observations such as that pictured in Fig. 1(a) and aims to validate the model and demonstrate the source of the striated patterns.

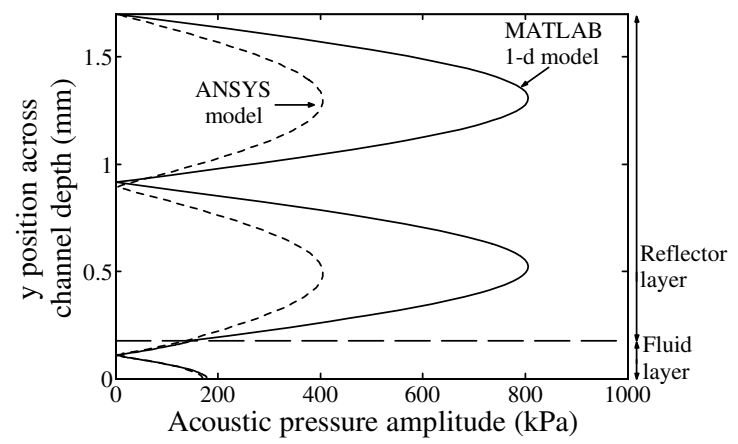

Fig. 3. Axial acoustic pressure fields predicted by the FEA and MATLAB models for resonant frequencies of $3.37 \mathrm{MHz}$ and $3.46 \mathrm{MHz}$ for the respective model, (based on an acoustic particle velocity of $0.002 \mathrm{~m} / \mathrm{s}$ at the fluid plane $y=0$ ).
The mode identified in the previous subsection $(\sim 3.4 \mathrm{MHz})$ is investigated as this is one of two modes which may be used during operation of the separator.

\section{Results and discussion}

\subsection{Analysis of test device}

Fig. 4(a) shows the results of an FE modal analysis of the reflector and fluid layers where white through to black contours indicate minimum to maximum pressure amplitude of the resonant mode. It can be seen that a standing wave of order approximately $m=2$ and $n=1$ is present in the reflector layer, which is predominantly a throughthickness resonance resembling the pressure profile in Fig. 3. The field pattern within the fluid layer is much more complex and is highly non-uniform, showing acoustic 'hot spots' at regular positions across the channel width. These hot spots suggest that particles will move to a series of lateral positions across the fluid chamber with an approximate spacing of $0.25 \mathrm{~mm}$. The experimentally observed striations are spaced at approximately $0.21 \mathrm{~mm}$ apart which is comparable to the modelled results. The small discrepancy between spacings may be due to inaccuracies in the fluid channel dimensions used in the simulation.

Based on the predicted pressure gradients, it is estimated that the lateral gradient of potential energy density (to which radiation force is proportional) may be as much as $85 \%$ of the more dominant axial gradient, measured in the central region of the fluid layer near the plane of symmetry. This suggests that radiation forces as a result of enclosure modes will significantly influence the trajectory of a particle and resulting separation performance of the device.

Lateral variations in the fluid layer acoustic field also cause variations in the axial strength of the radiation force which may influence the effectiveness of particle separation. The action of lateral radiation forces will induce particle agglomeration, such as the striations observed, allowing short range inter-particle forces to cause particles to cluster further and sediment out of suspension, consistent with observations.

\subsection{Influence of side-wall width}

The boundary conditions applied to a cavity have a bearing on the mode shape and resonant frequency. Therefore, it is likely that the side-wall of the fluid chamber formed by the Pyrex influences the lateral mode shape seen in the fluid layer, but by maintaining the $y$ structural characteristics the dominant through-thickness resonance remains relatively unaffected. In Fig. 4 it can be seen that when the thickness of the side-wall is decreased from $0.9 \mathrm{~mm}$ (a) to $0.2 \mathrm{~mm}$ (b) whilst retaining a fluid channel width of $5 \mathrm{~mm}$, the distinctive lateral variations lose definition and the field becomes more uniform across the width. Based on Fig. 4 and analyses using other wall thicknesses, 
(a)

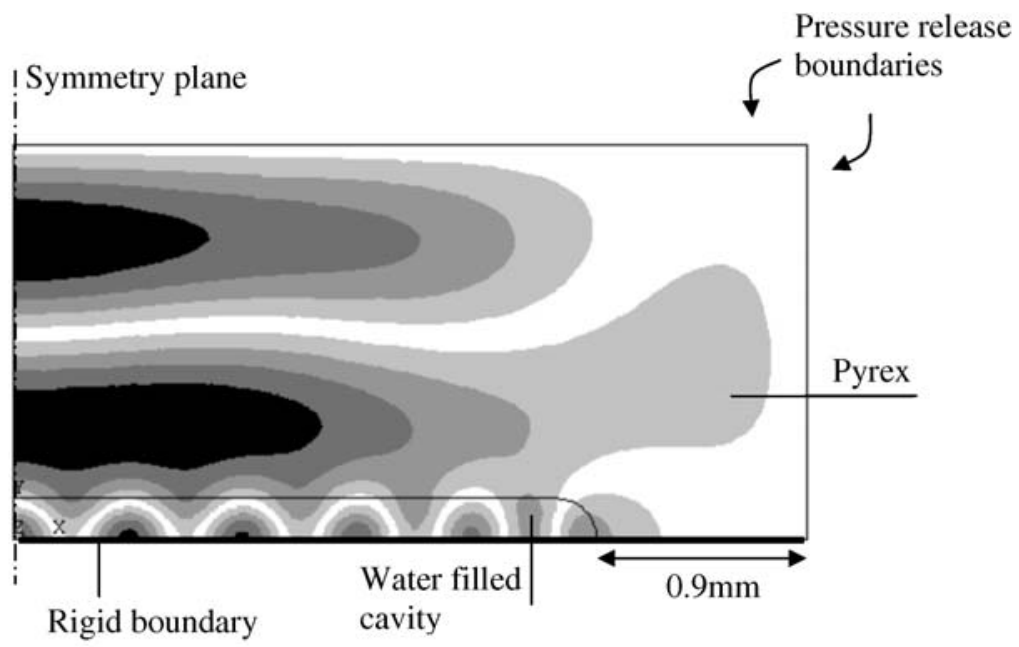

(b)

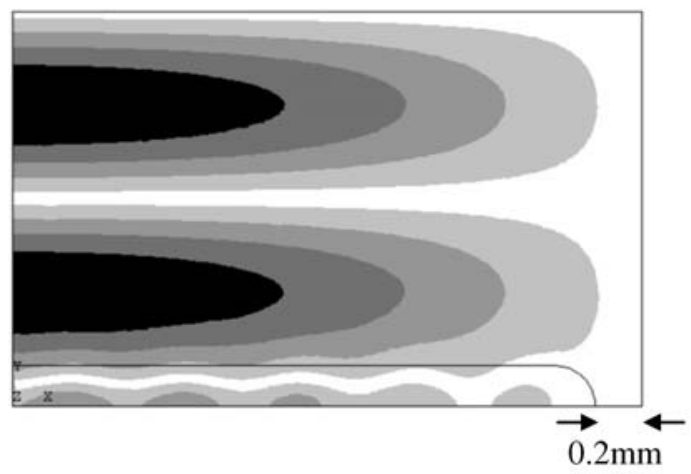

(c)

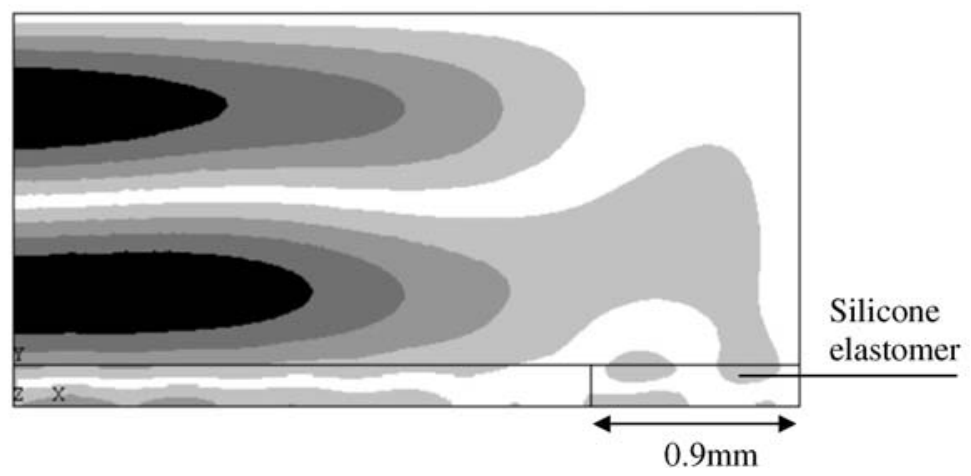

Fig. 4. Results of FEA modal analyses using (a) the geometry and materials of the fabricated test device and boundary conditions applied to the model, (b) reduced side-wall width and (c) alternative side-wall material (silicone elastomer). All simulations predict a resonant frequency of $3.4 \pm 0.01 \mathrm{MHz}$.

the magnitude of the lateral variations appears highly dependent on the impedance presented to the fluid by the side-wall. The lower acoustic impedance offered by the thinner side-wall results in a reduction in acoustic pressure at the fluid chamber/side-wall boundary and a more uniform lateral field.

In all three plots of Fig. 4, the acoustic pressure variations within the fluid layer increase in magnitude from the side-wall boundary towards the centre of the fluid layer (from right to left). Correspondingly, the magnitude of the axial $(y)$ acoustic radiation force will increase to a maxi- mum at the centre and therefore a particle will converge towards the nodal plane at a greater rate within the centre of the fluid layer. This is consistent with measurements of acoustic pressure in a similar device [8].

Generally the results suggest that by reducing the sidewall width, particles passing through the fluid layer will move towards a better defined nodal plane across the fluid layer width, rather than moving to acoustic hot spots and forming striations. In addition it may be possible to tune the performance of existing devices by the removal of wall material. 


\subsection{Influence of side-wall material}

Some related devices rely on a separate spacer layer to define the fluid layer depth and use a seal to form the side-walls $[8,9]$. In these devices, lateral forces do not appear to be as significant. The use of an acoustically damping material, e.g. silicone elastomer Sylgard 182, to form the fluid layer side-walls may contribute to the suppression of lateral modes.

Fig. 4(c) illustrates the mode shape for the throughthickness resonant frequency, but replacing the $0.9 \mathrm{~mm}$ wide Pyrex side-walls in Fig. 4(a) with a rectangular cross-section elastomer region. A significant difference between the fluid layer mode shapes of Fig. 4(a) and (c) can be seen with the latter, using the elastomer side-wall, not predicting the presence of acoustic hot spots across the width of the fluid layer and, although the contours suggest a small degree of variability in the acoustic field, it is significantly more uniform. The elastomer region appears to exhibit its own low amplitude enclosure mode as a quadrant pattern can be discerned.

The improved lateral uniformity of the acoustic field could be in part attributed to the properties of the elastomer material being more closely matched to the fluid, significantly altering the impedance boundary conditions of the fluid layer and mode patterns.

\section{Conclusions}

Within particle manipulation devices, lateral variations in the acoustic field can give rise to lateral radiation forces which significantly influence the trajectories of particles and cause particles to agglomerate at certain sites. Although acoustic interference and structural modes can give rise to lateral variations, this paper demonstrates that acoustic enclosure modes are a likely cause within the separator device and should be investigated further.

The model predicts that due to enclosure modes acoustic 'hot spots' exist across the width of the fluid layer, their location corresponding with particle striations observed in experiment. The simulations also predict that excitation of enclosure modes can give rise to lateral forces which are of the same order of magnitude as the axial forces. FEA is presented as a useful tool to investigate the influence of geometric and material variations upon the acoustic field. Further development of the model will allow near-field and structural modes to be simulated.

\section{Acknowledgements}

This work was funded through EPSRC grant GR/ R13333/01. The authors also gratefully acknowledge dstl and Porvair Plc. for their support.

\section{References}

[1] S.M. Woodside, J.M. Piret, M. Groschl, E. Benes, B.D. Bowen, Acoustic force distribution in resonators for ultrasonic particle separation, AIChE Journal 44 (1998) 1976-1984.

[2] T. Lilliehorn, U. Simu, M. Nilsson, M. Almqvist, T. Stepinski, T. Laurell, J. Nilsson, S. Johansson, Trapping of microparticles in the near field of an ultrasonic transducer, Ultrasonics 43 (2005) 293303.

[3] A. Haake, A. Neild, D.H. Kim, J.E. Ihm, Y. Sun, J. Dual, B.K. Ju, Manipulation of cells using an ultrasonic pressure field, Ultrasound in Medicine and Biology 31 (2005) 857-864.

[4] N.R. Harris, M. Hill, S. Beeby, Y. Shen, N.M. White, J.J. Hawkes, W.T. Coakley, A silicon microfluidic ultrasonic separator, Sensors and Actuators B-Chemical 95 (2003) 425-434.

[5] L.E. Kinsler, A.R. Frey, A.B. Coppens, J.V. Sanders, Fundamentals of Acoustics, third ed., Wiley, New York, 1982.

[6] M. Hill, R.J.K. Wood, Modelling in the design of a flow-through ultrasonic separator, Ultrasonics 38 (2000) 662-665.

[7] M. Hill, Y.J. Shen, J.J. Hawkes, Modelling of layered resonators for ultrasonic separation, Ultrasonics 40 (2002) 385-392.

[8] S.P. Martin, R.J. Townsend, L.A. Kuznetsova, K.A.J. Borthwick, M. Hill, M.B. McDonnell, W.T. Coakley, Spore and micro-particle capture on an immunosensor surface in an ultrasound standing wave system, Biosensors \& Bioelectronics. 21 (2005) 758-767.

[9] J.J. Hawkes, M.J. Long, W.T. Coakley, M.B. McDonnell, Ultrasonic deposition of cells on a surface, Biosensors \& Bioelectronics 19 (2004) $1021-1028$ 